\title{
Perspectives d'utilisation de la pervaporation dans le traitement de l'alcool de fermentation
}

\author{
par \\ Q T. NGUYEN, R. CLEMENT et J. NEEL
}

\section{R és u m é}

Les différentes possibilités d'utilisation de la pervaporation dans la production de l'alcool anhydre à partir du milieu de fermentation ont été envisagées. La voie la plus intéressante serait l'extraction de l'alcool sous forme pure à travers une membrane infiniment sélective vis-à-vis de l'éthanol. Cependant, une telle pellicule n'existe pas encore et sa mise au point semble très difficile dans l'état actuel des connaissances sur les membranes.

L'enrichissement en alcool est envisageable avec certaines parois sélectives à l'alcool disponibles sur le marché mais leur sélectivité n'est pas suffisante pour permettre un enrichissement en une seule étape jusqu'à une teneur de l'ordre de $80 \%$. Par contre, un mélange à cette composition serait économiquement déshydraté à l'aide des membranes existantes.

Le film actuellement le plus performant permettrait une déshydratation de 10 à $0,5 \%$ en teneur aqueuse avec un coût énergétique de l'ordre de $320 \mathrm{KJ} / \mathrm{Kg}$ d'éthanol pur.

La déshydratation de l'alcool à $80 \%$ obtenu par distillation serait aussi une option intéressante $(750 \mathrm{KJ} / \mathrm{Kg}$ d'alcool anhydre).

Enfin, on pourrait envisager une régulation en continu de la teneur en alcool dans le fermenteur à une concentration optimale par pervaporation avec une membrane sélective à l'alcool. Cependant, une distillation intermédiaire serait nécessaire, car l'on ne dispose pas

Laboratoire de chimie-physique macromoléculaire, C.N.R.S. - E.R.A. 23 E.N.S.I.C., 1, rue Grandville - 54042 Nancy cedex. 
encore de parois suffisamment sélectives dans la gamme des concentrations intermédiaires.

Mots clés : Energie - Séparation - Pervaporation - Ethanol de fermentation Membranes.

\title{
S u m m a r y
}

\author{
PROSPECTIVE USES OF PERVAPORATION \\ IN THE TREATMENT OF FERMENTATION ALCOHOL
}

Pervaporation is a membrane separation process for liquid mixtures which consists in selectively vaporizing a part of a mixture through a dense polymeric membrane by means of a vacuum or an inert gas stream. This is made possible by the selective sorption of the polymeric film, of which one side is in contact with the liquid and the other in contact with a gas phase of low vapor pressure.

In this paper, we envisage different process schemes involving pervaporation for the production of anhydrous alcohol from the fermentation medium. The most interesting way would be the extraction of alcohol in the pure form through a membrane infinitely selective to alcohol. However, such a film does not yet exist and its manufacture seems to be very problematical in the present state of membrane science.

The enrichment in alcohol using some available alcohol selective barriers can be envisaged, but their performance is not good enough to allow a one-step enrichment from $5 \mathrm{wt} \%$ to about $80 \mathrm{wt} \%$ alcohol.

Existing membranes would be sufficient to dehydrate a mixture at $80 \%-90 \%$ alcohol economically. The most efficient membrane presently existing would allow dehydration from $90 \%$ to $99.5 \%$ alcohol with an energy requirement of about $320 \mathrm{KJ}$ per kilogramme of pure alcohol.

Finally, one could a continuous extraction of alcohol from the fermenter by pervaporation in order to increase the yield of sugar conversion. Nevertheless, a distillation step would still be necessary as there is no good membrane adapted to this intermediate concentration range.

The energy requirements of different process schemes are discussed and compared with those of the conventional distillation process.

Key words: Energy - Pervaporation - Separation - Fermentation Alcohol Membranes. 


\section{INTRODUCTION}

A l'heure actuelle, la production d'alcool anhydre à partir de produits carbohydratés ou de résidus agricoles constitue dans un certain nombre de pays une option à court terme pour augmenter l'approvisionnement en carburant liquide. Cependant, plusieurs problèmes restent à résoudre pour qu'une mise en œuvre à grande échelle soit envisagée. Un des problèmes concerne les coûts de séparation et de purification de l'éthanol à partir des mélanges aqueux et dilués résultant de la fermentation.

Dans le procédé classique, le milieu de fermentation qui contient de 5 à $10 \%$ en alcool est distillé sous pression atmosphérique après clarification. La distillation sous pression atmosphérique produit un azéotrope contenant environ $5 \%$ d'eau. Pour être utilisable dans le carburol (mélange d'essence et d'alcool éthylique), cet alcool doit être déshydraté par distillation en présence d'un tiers constituant jusqu'à une pureté supérieure à 99,5\% (en pourcentages massiques).

On estime que la technique de purification conventionnelle nécessite une quantité de l'ordre de $4900 \mathrm{kcal}$ d'énergie primaire par kilo d'alcool (Metha, 1982). Ainsi, la seule étape de séparation nécessite environ $75 \%$ de l'énergie de combustion potentielle contenue dans l'alcool. Or, il faut ajouter l'énergie nécessaire pour produire et transporter les matières premières et pour leur fermentation, sans parler du coût de condensation. La mise au point de procédés moins gourmands en énergie semble donc indispensable au développement de cette source énergétique.

L'utilisation des procédés à membranes pour la séparation et la purification d'éthanol a été proposée et des recherches exploratoires ont été entreprises. Parmi les procédés à membranes, la pervaporation apparaît a priori comme le plus adapté, car il s'agit d'un procédé de fractionnement de mélanges de solvants dans lequel la pression osmotique n'est pas un paramètre limitant, à la différence de ce qui est observé en osmose inverse (Metha, 1982). Dans cet article, nous nous proposons d'évaluer les possibilités de cette technique dans la séparation et la purification de l'alcool de fermentation.

\section{LA PERVAPORATION}

La pervaporation est un procédé qui permet de fractionner en continu des mélanges liquides généralement binaires. Le mélange brut A-B est mis en contact avec un film organique choisi à cause de son affinité préférentielle avec le constituant minoritaire A. Tandis que le perméat produit à la face aval de la membrane est continuellement évacué sous forme vapeur, soit à l'aide d'un balayage gazeux, 
soit par le maintien d'une pression réduite, le rétentat accumulé sur la face amont est principalement constitué du composé majoritaire purifié.

Ce procédé est donc, contrairement aux autres techniques utilisant des membranes, un procédé dans lequel les constituants qui sont permés subissent un changement d'état.

En choisissant correctement la nature du film séparateur, on peut obtenir, à une composition donnée, des sélectivités de partage supérieures à celle qui peut être réalisée par un simple étage de distillation. Ce qui, en conséquence, diminue d'autant la quantité d'énergie requise pour assurer l'épuration. En outre, la composition pour laquelle la membrane n'est plus sélective est généralement très différente de la composition azéotropique.

Enfin, le procédé de pervaporation ne demande pour l'essentiel que des calories qui peuvent être prélevées sur une source de bas niveau énergétique $\left(25^{\circ}-100^{\circ} \mathrm{C}\right)$, ce qui permet également de l'envisager pour le traitement d'espèces chimiquement thermolabiles. Tous ces faits présentent donc la pervaporation comme une technique riche en possibilités; ainsí, en supposant que l'on puisse disposer d'une membrane présentant une sélectivité infinie pour l'espèce minoritaire dans un mélange, c'est-à-dire une membrane qui ne laisse passer que cette espèce, l'obtention du produit à l'état pur ne coûterait que son énergie de vaporisation. Rappelons qu'en osmose inverse, c'est toujours le constituant majoritaire qui est extrait à travers la membrane, et qu'en distillation c'est la nature chimique du mélange qui impose le produit majoritaire dans le distillat.

Cependant, bien que la pervaporation soit étudiée depuis plus de 25 ans (Binning, 1958), elle commence à peine à entrer dans la réalité industrielle. Cette lenteur de développement est due à deux faits essentiels :

- chaque famille de mélanges demande la mise au point de membranes spécifiques;

- le transfert transmembranaire est un processus lent qu'il faut compenser par des investissements dans des modules comportant de grandes surfaces actives.

\subsection{Paramètres de fonctionnement de la pervaporation}

Le film membranaire est caractérisé par deux paramètres qui sont, d'une part la sélectivité $\beta$, et le flux de pervaporat $\mathrm{J}_{\mathrm{p}}$ d'autre part.

Pour un mélange de composition donnée, la sélectivité $\beta$ (c) représente le rapport des teneurs pondérales c' et c en constituant A (minoritaire et migrant préférentiellement) dans la pervaporat et dans le rétentat d'origine respectivement, soit : $\beta$ (c) $=\mathrm{c}^{\prime} / \mathrm{c}$.

Cette grandeur, tout comme le flux $\mathrm{J}_{\mathrm{p}}(\mathrm{c})$ sont donc des paramètres dont les amplitudes varient avec la composition du mélange liquide 


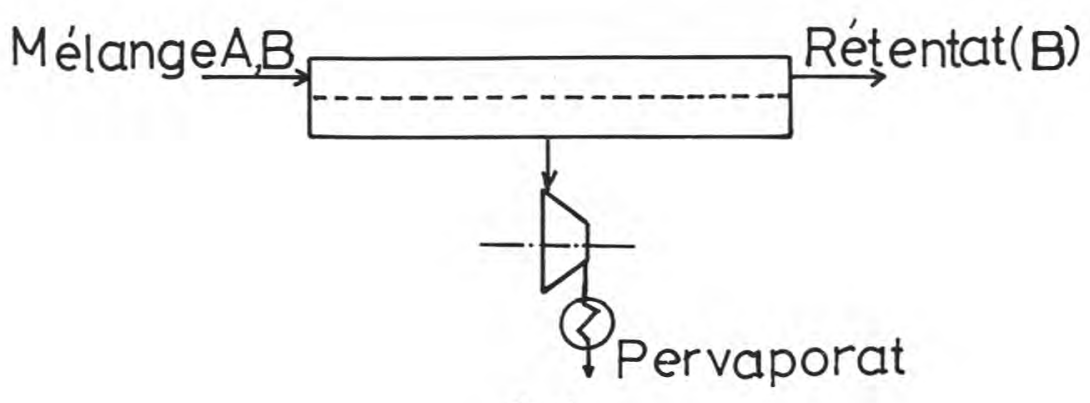

fig. 1

Principe de la pervaporation. - Principle of pervaporation.

en contact avec la membrane. Il est ainsi nécessaire d'établir expérimentalement une correspondance entre la composition du mélange à traiter et les valeurs ponctuelles des paramètres de fonctionnement. Ce qui permet ensuite d'exprimer ceux-ci sous forme de fonctions mathématiques empiriques utilisables pour réaliser la modélisation du procédé.

Une membrane sera d'autant plus avantageuse du point de vue énergétique qu'elle tendra à limiter strictement la quantité de matière vaporisée à l'espèce minoritaire dans le mélange. En conséquence, la recherche de membranes possédant de bonnes sélectivités doit être l'objectif prioritaire pour limiter le coût du fonctionnement. En revanche, pour limiter le coût d'investissement en surface membranaire et modules associés, le flux de pervaporat doit être le plus élevé possible. Or, ces deux grandeurs varient généralement en sens inverse. Nous avons mis en évidence (voir l'annexe) un critère de performance, relatif à la production, qui tient compte de ces paramètres antagonistes. Il nous permettra de chiffrer la qualité d'une membrane vis-à-vis d'un mélange de composition donnée.

\section{SCHEMAS POSSIBLES DE MISE EN OEUVRE DU PROCEDE DE PERVAPORATION APPLIQUE A LA PURIFICATION DE L'ETHANOL}

\subsection{Fonctionnement idéal}

Comme cela a été dit précédemment, la pervaporation ne peut fonctionner dans des conditions énergétiquement favorables que si le constituant minoritaire d'un mélange est extrait avec la plus grande sélectivité et le plus grand débit possibles. 
Afin de traiter le milieu de fermentation, le problème se pose donc de disposer d'une membrane qui ne laisse pratiquement pas passer l'eau, mais qui soit néanmoins relativement bien perméable à l'alcool éthylique. Si une telle membrane existait, le coût de déshydratation de l'éthanol serait réduit à la dépense énergétique correspondant à la séparation de ce dernier, soit $920 \mathrm{KJ}$. Cette dépense ne représenterait alors qu'environ 3,5\% du potentiel énergétique contenu dans le carburant obtenu.

Malheureusement, dans l'état actuel des connaissances, il semble très peu probable d'obtenir une telle membrane. En effet, les propriétés physico-chimiques du système eau-alcool rendent très difficile la résolution de ce problème : un matériau ayant une forte affinité pour l'alcool laissera également passer une proportion élevée d'eau à cause de l'existence d'interactions fortes (liaisons hydrogènes) entre les deux constituants. Pour empêcher le passage de l'eau, on peut choisir une membrane très hydrophobe afin de profiter du caractère hydrophobe plus marqué, dû au radical éthyle, de l'éthanol. Mais, même dans ce cas, l'eau réussirait néanmoins à traverser partiellement une telle paroi à cause de sa faible dimension moléculaire (qui favorise sa diffusivité).

Il n'est donc pas envisageable de n'utiliser que ce seul type de membrane pour purifier l'éthanol de fermentation. L'utilisation de la pervaporation appliquée à cette tâche requiert soit l'usage successif de deux types de membranes complémentaires et/ou le couplage avec une autre technique de séparation. Parmi les techniques de séparation de deux solvants, la distillation apparaît comme la plus intéressante pour l'enrichissement des solutions à faible teneur d'alcool, et ceci jusqu'à des teneurs de $80 \%$ environ. En effet, dans ce domaine de concentrations, la phase vapeur, issue du liquide, est très enrichie en alcool relativement à son milieu d'origine (fig. 2), ce qui permet une distillation à faible reflux, donc à faible coût énergétique. Notons que dans tout ce qui va suivre, toutes les teneurs en alcool sont exprimées en pourcentage massique (pondéral).

\subsection{Performances des membranes actuelles vis-à-vis de la séparation eau-éthanol}

Le champ d'application de la pervaporation étant plutôt limité à l'épuration d'un mélange par migration transmembranaire du polluant, cette technique est donc susceptible d'être appliquée aux deux domaines extrêmes de concentrations en alcool, à savoir : extraction d'alcool de solutions à faibles teneurs ou déshydratation de mélanges titrant bien plus de $50 \%$.

En outre, les performances citées ci-après ne constituent en aucune façon une limite supérieure des possibilités de la technique, mais sont le reflet actuel de l'état d'avancement des recherches appliquées au problème de séparation envisagé. 


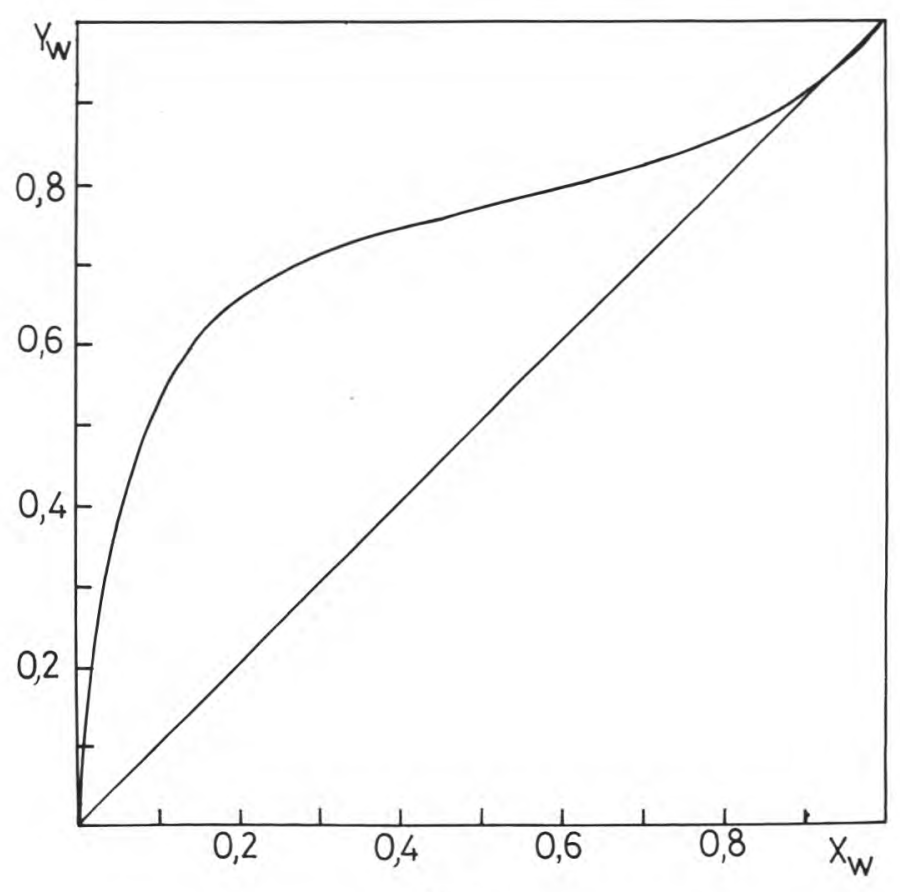

fig. 2

Courbe d'équilibre liquide-vapeur pour eau-alcool sous $1 \mathrm{~atm}$.

Teneur en alcool en phase vapeur versus teneur en alcool en phase liquide.

Liquid-vapor composition curve for water-ethanol mixtures under $1 \mathrm{~atm}$.

Ethanol content in vapor phase versus ethanol content in liquid phase.

\subsubsection{Extraction d'alcool d'un milieu aqueux dilué}

Il existe actuellement deux natures de film qui présentent des sélectivités notables en pervaparation. Il s'agit, d'une part, de la membrane FT 30 produite par Film Tec Corp. (Schissel et Orth, 1984), et de membranes à base de silicones d'autre part, dont la plus performante a été étudiée par Hoover et Hwang (1982). Cette dernière, qui est la plus sélective, ne conduit cependant pas à des pervaporats sensiblement plus riches en alcool que les phases vapeur que l'on peut obtenir par distillation simple sous pression atmosphérique. La figure 3 montre la sélectivité comparée des deux membranes citées avec la simple vaporisation.

Alors que la distillation permet de réaliser plusieurs équilibres liquides-vapeur en cascade dans la même colonne par le biais du reflux interne, le fonctionnement d'une cascade de pervaporateurs nécessite d'intercaler entre chaque module une unité de recompres- 


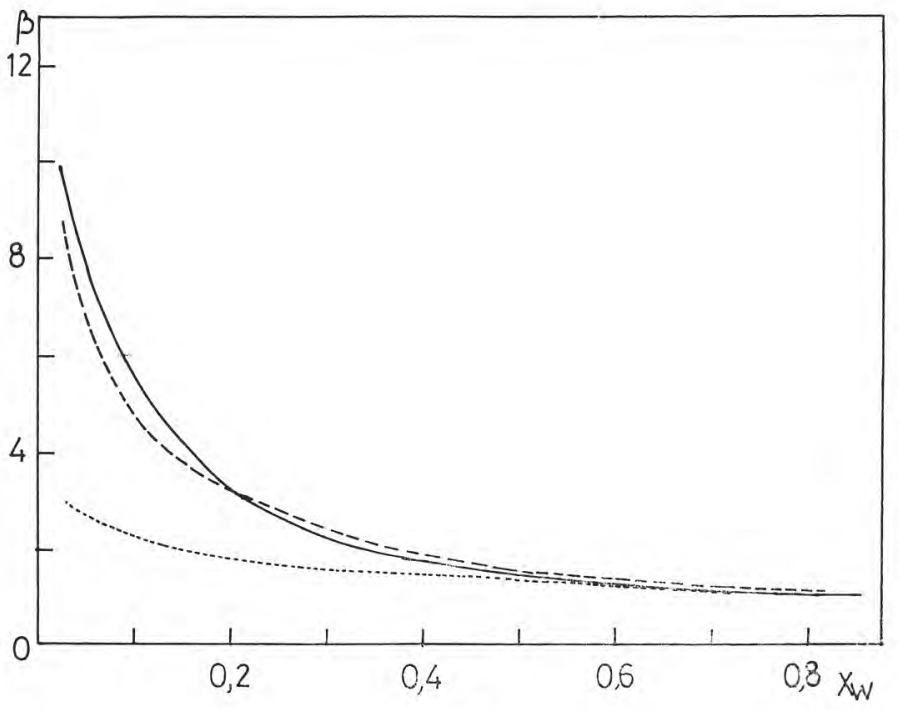

fig. 3

Sélectivités comparatives des membranes Silicones (-.-), FT 30 (...) et de la vaporisation ( $\longrightarrow$ ) vis-à-vis du mélange eau- alcool en fonction de la composition du mélange.

Comparison of selectivity of the Silicone (--)and FT30 (...) membranes and vaporization $(\longrightarrow)$ for the water-ethanol mixtures in function of the composition of the mixture.

sion-condensation, qui alourdit relativement ce dernier procédé. Or, la concentration en alcool extrait dans le pervaporat étant fixée par les caractéristiques de la membrane et leurs évolutions avec les conditions opératoires, on est obligé, en fonction des performances des membranes actuelles, de recourir au dispositif en cascade pour atteindre la teneur désirée en alcool.

Le tableau 1 donne quelques résultats de simulation de concentration de mélanges à faibles teneurs en alcool obtenues à partir des données de Hoover et Hwang (1982). En particulier le traitement d'alcool à $6 \%$ pour le ramener à $3 \%$ y est présenté. Dans ces conditions, le perméat extrait ne contiendrait que $32,4 \%$ d'alcool. Si on limitait la plage de fonctionnement de la membrane au voisinage de la composition de départ, la teneur de la vapeur obtenue ne dépasserait pas $37 \%$, valeur qui correspond à la limite de sélectivité de la membrane en ce point. (La méthode de calcul est présentée en annexe). 


\section{TABLEAU $1-T A B L E 1$}

Enrichissement en alcool par pervaporation. Performances et coûts énergétiques concernant la membrane en Silicone. Calculs faits à partir des données de Hoover et Hwang, 1982. Les énergies sont données sur la base de $1 \mathrm{~kg}$ d'alcool anhydre produit

Enrichment in alcohol by pervaporation. Performances and energetic costs concerning the Silicone membrane as calculated from data of Hoover and HWANG, 1982. The energy requirements are stated per kilogram of anhydrous alcohol product.

\begin{tabular}{|c|c|c|c|c|c|}
\hline \multicolumn{3}{|c|}{$\begin{array}{c}\text { Teneurs pondérales } \\
\text { en alcool } \\
(\mathrm{Kg} / \mathrm{Kg})\end{array}$} & \multicolumn{2}{|c|}{$\underset{\left(\mathrm{Kg} \cdot \mathrm{h}^{-1} \cdot \mathrm{m}^{-2}\right.}{\text { Flux }}$} & \multirow{2}{*}{$\begin{array}{c}\text { Energie } \\
\mathrm{KJ} / \mathrm{Kg} \mathrm{d} \text { alcool pur } \\
\text { dans le pervaporat }\end{array}$} \\
\hline $\begin{array}{c}\text { Mélange } \\
\text { initial }\end{array}$ & Rétentat & $\begin{array}{l}\text { Perva- } \\
\text { porat }\end{array}$ & $\begin{array}{l}\text { Perva- } \\
\text { porat }\end{array}$ & $\begin{array}{c}\text { Traite- } \\
\text { ment }\end{array}$ & \\
\hline 0,06 & 0,03 & 0,324 & 0,37 & 3,62 & 6030 \\
\hline 0,324 & 0,06 & 0,651 & 0,33 & 0,75 & 2230 \\
\hline 0,05 & 0,005 & 0,254 & 0,38 & 2,09 & 8130 \\
\hline
\end{tabular}

\section{TABLEAU $2-T A B L E 2$}

Déshydratation d'alcool par pervaporation.

Performances et coûts énergétiques concernant la membrane G.F.T. calculés à partir des données de Soukup, 1983

Dehydration of alcohol by pervaporation.

Performances and energetic costs concerning the G.F.T. membrane as calculated from data of Soukup, 1983

\begin{tabular}{c|c|c|c|c}
\hline \multicolumn{2}{c|}{$\begin{array}{c}\text { Teneurs pondérales } \\
\text { en eau } \\
(\mathrm{Kg} / \mathrm{Kg})\end{array}$} & $\begin{array}{c}\text { Energie } \\
\text { (KJ/Kg dalcool pur } \\
\text { dans le rétentat) }\end{array}$ & $\begin{array}{c}\text { Rendement } \\
\text { en alcool } \\
\%\end{array}$ \\
\hline $\begin{array}{c}\text { Mélange } \\
\text { initial }\end{array}$ & Rétentat & $\begin{array}{c}\text { Perva- } \\
\text { porat }\end{array}$ & 125 & 99,3 \\
\cline { 3 - 5 } 0,05 & 0,005 & 0,88 & 270 & 99,2 \\
0,10 & 0,005 & 0,93 & 625 & 98,3 \\
0,20 & 0,005 & 0,94 & 1360 & 97,4 \\
0,35 & 0,005 & 0,954 & & \\
\hline
\end{tabular}




\subsubsection{Déshydratation de l'alcool}

Il existe actuellement sur le marché plusieurs membranes qui pourraient permettre d'extraire efficacement l'eau d'un mélange riche en alcool. La meilleure membrane serait celle fabriquée par G.F.T. et étudiée par Soukup, 1983 (voir tab. 2). Dans ces conditions, il serait doublement avantageux d'utiliser la pervaporation pour déshydrater l'alcool. Tout d'abord, la sélectivité est faible pour la distillation dans ce domaine de concentration, tandis que l'on extrait un perméat à plus de $90 \%$ en eau à travers les meilleures membranes. Ensuite, le rétentat liquide est constitué d'alcool pratiquement pur dont on peut très facilement ajuster la teneur en fixant la surface de membrane (ou le temps de séjour). De plus, la déshydratation pourrait se faire en une seule étape et à faible coût énergétique. Rappelons qu'au voisinage de la composition sub-azéotropique (entre 90 et $95,5 \%$ d'alcool), la sélectivité de la distillation est très faible, ce qui implique une consommation énergétique élevée au niveau du bouilleur pour enlever $5 \%$ d'eau du mélange. En outre, dans la zone post-azéotropique, le recours nécessaire à la distillation azéotropique ternaire est également onéreux à cause de la proportion importante du tiers constituant qu'il faut vaporiser (Black, 1980).

\subsection{Calcul énergétique des procédés faisant intervenir la pervaporation}

\subsubsection{Procédé « tout membrane » (schéma 1, fig. 4)}

Dans ce procédé, deux étages de pervaporateurs utilisent une membrane favorable à l'alcool (membrane en silicones) et sont couplés

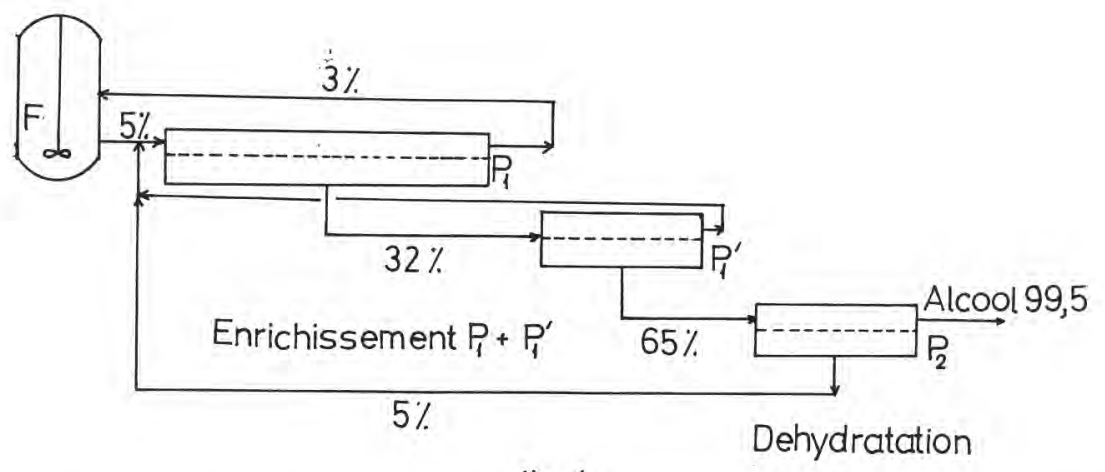

fig. 4

Schéma de procédé $n^{\circ} 1$. Couplage de 2 étages de pervaporateurs $P_{1}$ et $P_{1}^{\prime}$ (pour l'enrichissement en alcool) avec un étage de déshydratation $\mathrm{P}_{2}$.

Process scheme $n^{\circ} 1$. Coupling of two stages of $P_{I}$ and $P_{1}^{\prime}$ pervaporators (for alcohol enrichment) with a dehydrating pervaporator $P_{2}$. 
Coûts énergétiques de différents procédés de production d'alcool anhydre

$(\mathrm{a}+\mathrm{b})$ Ces valeurs sont estimées à partir des résultats de calculs de BLACK, 1980 (a) et des données de distillation réelle (b, Muller et DuquenNoy, 1982).

(c) L'énergie potentielle de combustion de l'éthanol est prise égale à $29673 \mathrm{KJ} / \mathrm{Kg}$ (BLACK, 1980).

(d) Valeur moyenne couramment admise.

Energetic costs of different processes for pure alcohol production

$(\mathrm{a}+\mathrm{b})$ These values are estimated from the results of BLAcK's calculation (1980) and from the data on the actual distillation operation (MULLER and DUQUENNOY, 1982).

(c) From the heat of combustion of $29670 \mathrm{KJ} / \mathrm{Kg}$ for ethanol (BLACK, 1980).

(d) Average value usually admitted.

\begin{tabular}{|c|c|c|c|c|c|}
\hline \multirow{2}{*}{$n^{\circ}$} & \multicolumn{3}{|c|}{$\begin{array}{l}\text { Energie des étapes du procédé } \\
\text { (KJ/Kg d'alcool pur dans le produit) }\end{array}$} & \multirow{2}{*}{$\begin{array}{l}\text { Energie totale } \\
\text { requise pour le trai- } \\
\text { tement de } 5 \% \text { à } \\
99,5 \% \text { en alcool }\end{array}$} & \multirow{2}{*}{$\begin{array}{l}\text { Fraction de l'énergie } \\
\text { de combustion } \\
\text { d'alcool } \\
\text { (c) }\end{array}$} \\
\hline & Première étape & Deuxième étape & Troisième étape & & \\
\hline 1 & $\begin{array}{c}\text { Pervaporation } \\
5 \%-32 \% \\
9100 \text { thermique } \\
2070 \text { électrique }\end{array}$ & $\begin{array}{l}\text { Pervaporation } \\
32 \%-65 \% \\
3600 \text { thermique } \\
690 \text { électrique }\end{array}$ & $\begin{array}{c}\text { Pervaporation } \\
65 \%-99,5 \% \\
1360 \text { thermique } \\
170 \text { électrique }\end{array}$ & $\begin{array}{l}17090 \text { dont } 17 \% \text { en } \\
\text { énergie électrique }\end{array}$ & 0,576 \\
\hline 2 & $\begin{array}{c}\text { Pervaporation } \\
5 \%-34 \% \\
5680 \text { thermique } \\
1300 \text { électrique }\end{array}$ & $\begin{array}{l}\text { Distillation } \\
34 \%-90 \% \\
8000 \text { thermique } \\
220 \text { électrique } \\
(\mathrm{a}+\mathrm{b})\end{array}$ & $\begin{array}{c}\text { Pervaporation } \\
90 \%-99,5 \% \\
270 \text { thermique } \\
50 \text { électrique }\end{array}$ & $\begin{array}{l}15520 \text { dont } 10 \% \text { en } \\
\text { énergie électrique }\end{array}$ & 0,523 \\
\hline 3 & \multicolumn{2}{|c|}{$\begin{array}{l}\text { Distillation } 5 \text { - } 90 \% \\
8940 \text { thermique (b) } \\
245 \text { électrique (b) }\end{array}$} & $\begin{array}{l}\text { Pervaporation } \\
90 \%-99,5 \% \\
270 \text { thermique } \\
50 \text { électrique }\end{array}$ & $\begin{array}{l}9500 \text { dont } 3,4 \% \text { en } \\
\text { énergie électrique }\end{array}$ & 0,320 \\
\hline 4 & $\begin{array}{l}\text { Distillation } \\
5 \%-90 \% \\
8940 \text { thermique } \\
245 \text { électrique (b) }\end{array}$ & $\begin{array}{l}\text { Distillation } \\
90 \%-95 \% \\
9930 \text { thermique } \\
\text { (b) }\end{array}$ & $\begin{array}{c}\text { Distillation azéot. } \\
95 \%-99,5 \% \\
3400 \text { thermique } \\
\text { (d) }\end{array}$ & $\begin{array}{l}22510 \text { dont } 1,1 \% \text { en } \\
\text { énergie électrique }\end{array}$ & 0,760 \\
\hline 5 & $\begin{array}{l}\text { Distillation } \\
\text { avec RMV* } \\
5590 \text { thermique } \\
800 \text { électrique (b) }\end{array}$ & $\begin{array}{l}\text { Distillation } \\
\text { avec RMV* } \\
4950 \text { thermique } \\
860 \text { électrique (b) }\end{array}$ & $\begin{array}{l}\text { Distillation } \\
\text { azéotropique } \\
3400 \text { thermique } \\
\text { (d) }\end{array}$ & $\begin{array}{l}15600 \text { dont } 10,6 \% \text { en } \\
\text { énergie électrique }\end{array}$ & 0,526 \\
\hline
\end{tabular}


à un étage de déshydratation. Les deux premiers amenant le pervaporat à une teneur de $65 \%$ en alcool qui est directement déshydratée. Dans ce schéma, la consommation énergétique est élevée, principalement à cause de la faible sélectivité de la membrane en silicones, qui rend nécessaire l'utilisation de deux étages et le recyclage d'une partie non négligeable des solvants (tab. 3).

On peut raisonnablement espérer la mise au point dans un avenir proche de membranes plus électives à l'alcool, auquel cas un possible enrichissement en une seule étape de l'alcool jusqu'à 65-70 \% rendrait ce procédé très attrayant sur le plan énergétique.

\subsubsection{Procédé combinant une première extraction d'alcool par pervaporation, \\ une distillation et une déshydratation par pervaporation} (schéma 2, fig. 5)

Le but de ce procédé serait moins de réduire la consommation énergétique que d'extraire en continu l'alcool, permettant ainsi de pousser au maximum le rendement de conversion des sucres fermentescibles. En effet, il est bien connu que la fermentation alcoolique est un processus inhibé par le produit. En pervaporant l'alcool au fur et à mesure qu'il est libéré on maintiendrait la fermentation à un rythme acceptable sans augmenter trop le coût de séparation ultérieure. La deuxième étape préconisée est la distillation, car elle

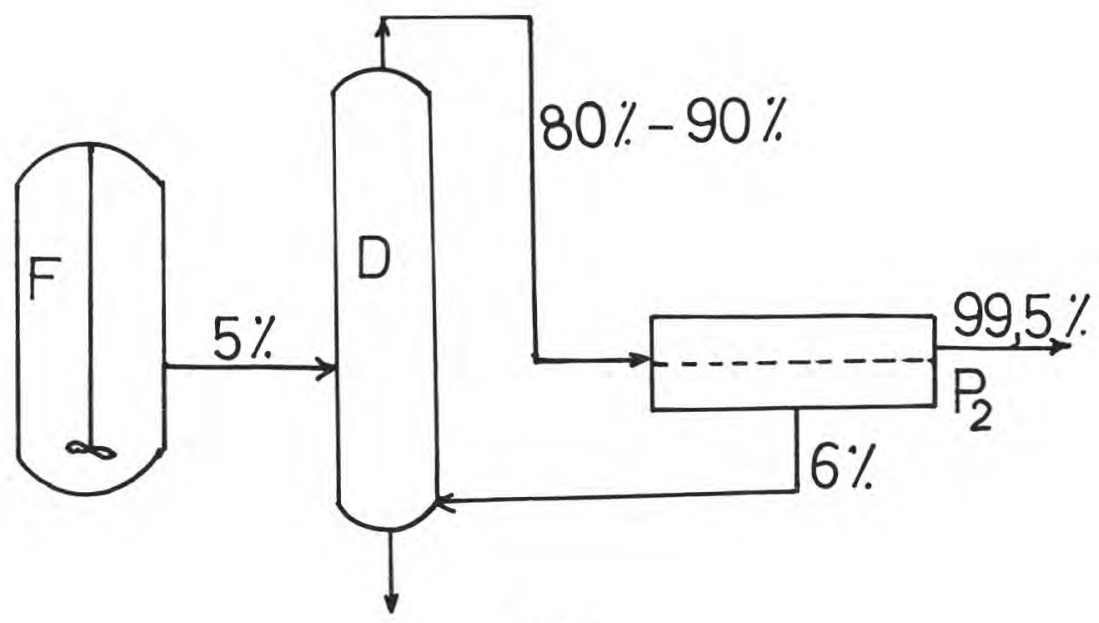

fig. 5

Schéma $\mathrm{n}^{\circ}$ 2. Couplage d'un pervaporateur $\mathrm{P}_{1}$ avec une colonne à distiller $\mathrm{D}$ et un pervaporateur de déshydratation $\mathrm{P}_{2}$.

Scheme $n^{\circ}$ 2. Coupling of a $P_{1}$ pervaporator with a distillation column $D$ and a dehydrating pervaporator $P_{2}$. 
est plus efficace que la pervaporation. A noter que ce procédé est légèrement plus avantageux que le premier en énergie (tab. 3), et il serait particulièrement intéressant dans le cas d'une fermentation à micro-organismes immobilisés car on pourrait traiter directement le milieu de fermentation sans prétraitement coûteux.

\subsubsection{Procédé utilisant le couplage d'une colonne à distiller} et un pervaporateur de déshydratation (schéma 3, fig. 6)

L'association des deux techniques, chacune dans le domaine de concentration qui lui est favorable, serait certainement le schéma le plus économique en énergie. Nous avons donné (tab. 3) les résultats du calcul énergétique concernant le cas d'une distillation jusqu'à $90 \%$. Or, il n'est pas évident que cette limite corresponde à un optimum énergétique. Cependant, la déshydratation de 80 à 99,5 ne coûterait que $630 \mathrm{KJ}$ d'origine thermique par kilogramme d'alcool pur contenu (plus $96 \mathrm{KJ}$ d'origine électrique/Kg d'alcool anhydre nécessaire au pompage et à la recompression du pervaporat).

Il serait intéressant de connaître dans ce cas la dépense d'énergie requise pour la concentration d'une solution de 5 à $80 \%$ par distillation.

\section{DISCUSSION GENERALE}

Dans le tableau 3, nous donnons également la consommation énergétique de deux schémas de production, par distillation, d'alcool

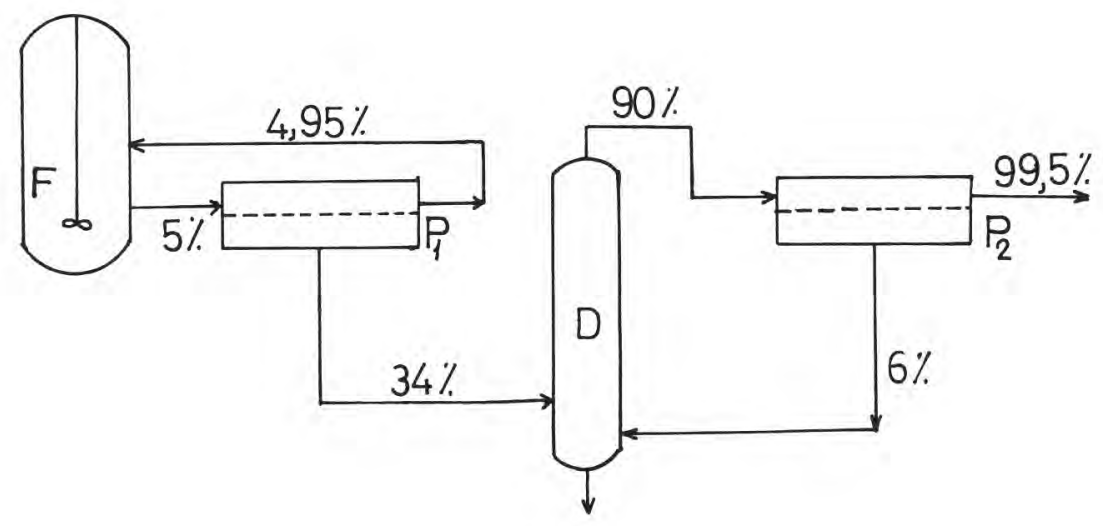

fig. 6

Schéma $\mathrm{n}^{\circ}$ 3. Couplage d'une colonne à distiller $\mathrm{D}$ avec un pervaporateur de déshydratation $\mathrm{P}_{2}$.

Scheme $n^{\circ}$ 3. Coupling of a distillation column $D$ with a dehydration pervaporator $P_{2}$. 
anhydre rapportés par Muller et Duquennoy, 1982. Il s'agit de valeurs de consommation réelles (celles des deux premières étapes viennent d'une distillerie française, celle concernant la déshydratation est la valeur moyenne recueillie par les auteurs de cet article auprès des industriels).

On y constate que, si la recompression mécanique a permis une économie notable, le rappart du coût énergétique de séparation de l'alcool au contenu énergétique du produit obtenu reste bien plus élevé que celui correspondant au $3^{e}$ schéma proposé. En outre, pour que la comparaison soit plus exacte il faudrait faire intervenir la valeur de la forme d'énergie utilisée. En effet, alors que l'énergie thermique utilisée en distillation est de la vapeur basse pression, donc coûteuse, des calories de bas niveau $\left(80^{\circ} \mathrm{C}\right)$ sont tout à fait suffisantes pour la pervaporation. Ces calories pourraient, par exemple, être récupérées à partir d'eaux chaudes qu'il faut actuellement refroidir avant rejet pour éviter la pollution thermique. De ce point de vue, la pervaporation est nettement plus intéressante que la distillation azéotropique. Par ailleurs, pour la partie déshydratation, la pervaporation est bien plus économique en énergie électrique que la distillation couplée avec recompression de vapeur, car la quantité d'énergie à récupérer dans la phase vapeur est moindre dans la première technique du fait que c'est principalement le constituant minoritaire qui est recomprimé.

Toujours pour la déshydratation, on peut citer l'étude de Leeper et Wankat (1982) sur l'extraction de l'alcool d'une solution à $90 \%$ par le gas-oil. Cependant, le chiffre indiqué pour la production du carburol, $6640 \mathrm{KJ} / \mathrm{Kg}$ d'alcool anhydre, nous semble trop optimiste.

\section{CONCLUSION}

En se fondant sur les résultats de cette étude, les conclusions suivantes peuvent être tirées :

1. La sélectivité des membranes actuellement connues, vis-à-vis de l'alcool en solution diluée, n'est pas suffisante pour rendre l'enrichissement par pervaporation compétitif avec la distillation. Néanmoins, l'extraction en continu de l'alcool du fermenteur pour diminuer l'inhibition des micro-organismes pourrait être intéressante. Si une membrane suffisamment sélective à l'alcool est mise au point, le procédé « toutes membranes " pourra devenir avantageux.

2. La déshydratation par membrane apparaît d'ores et déjà comme une solution très satisfaisante sur le plan énergétique. La simplicité du couplage distillation-pervaporation laisse présager une diminution de l'investissement. La teneur en alcool dans le distillat serait à optimiser en fonction des performances de la membrane et du taux de reflux réel dans la colonne à distiller. 
3. Un système de séparation à membrane est modulaire, donc peut être économiquement utilisé pour les petites et grandes installations, alors que l'efficacité (et aussi l'investissement) de systèmes modernes de distillation augmente avec la diminution en taille des unités.

\section{ANNEXE \\ Modélisation du procédé de pervaporation}

On considère un module tubulaire de périmètre $\mathrm{p}$, alimenté en mélange binaire, de teneur pondérale $\mathrm{C}_{0}$ en $\mathrm{A}$, avec un débit $\mathrm{Q}_{0}$, dont l'extérieur est maintenu sous pression réduite.

En admettant que l'écoulement interne est de type « piston », on se propose d'estimer les caractéristiques de production d'un tel module en composé $B$ épuré du constituant $A$ jusqu'à une teneur finale $\mathrm{C}_{\text {т. }}$.

\section{Mise en équation}

Faisons les bilans de matière relatifs à une tranche infinitésimale de longueur $\mathrm{dz}$ :

$$
\begin{aligned}
& \text { - bilan global : dQ }=-\mathrm{J}_{\mathrm{p}}(\mathrm{C}) \cdot \mathrm{p} \cdot \mathrm{dz} \text { (1); } \\
& \text { - bilan en constituant } \mathrm{A} \text { : } \\
& \mathrm{d}(\mathrm{Q} . \mathrm{C})=-\mathrm{J}_{\mathrm{p}}(\mathrm{C}) \cdot \mathrm{p} \cdot \mathrm{C} \cdot \beta(\mathrm{C}) \cdot \mathrm{dz} \\
& \text { relation qui, combinée avec (1), donne : } \\
& \text { Q.dc }=J_{\mathrm{p}}(\mathrm{C}) \cdot[1-\beta(\mathrm{C})] . C \cdot \mathrm{p} . \mathrm{dz}
\end{aligned}
$$

En considérant que l'énergie de vaporisation est entièrement apportée par le liquide et en négligeant la contribution de la chaleur de mélange, on peut également faire le bilan énergétique simplifié dans le petit élément considéré. $\mathrm{Si} \mathrm{C}_{\mathrm{p}}(\mathrm{C})$ et $\mathrm{L}_{\mathrm{p}}\left(\mathrm{C}^{\prime}\right)$ sont respectivement la capacité calorifique du liquide et la chaleur latente de vaporisation moyenne de la vapeur, l'énergie nécessaire à la vaporisation du pervaparat sera alors compensée par une chute thermique $\mathrm{d} \theta$, du liquide telle que :

$$
\text { Q.C } C_{\mathrm{p}}(\mathrm{C}) \cdot \mathrm{d} \theta=\mathrm{L}_{\mathrm{p}}\left(\mathrm{C}^{\prime}\right) . \mathrm{J}_{\mathrm{p}} \text { (C).p.dz (3). }
$$

\section{Caractéristiques de fonctionnement}

Les relations (1) à (3) constituent un système d'équations différentielles du premier ordre numériquement soluble dès que $\beta$ (C) et $\mathrm{J}_{\mathrm{p}}(\mathrm{C})$ sont connues. La résolution numérique de ce système permet d'associer à la distance, $\mathrm{z}$, parcourue dans le module les valeurs de $\mathrm{Q}, \mathrm{C}$ et $\Delta \theta$ correspondantes.

On en déduit directement les caractéristiques d'un module de surface unitaire assurant une épuration jusqu'à une teneur résiduelle $\mathrm{C}_{\text {т. }}$. 
- capacité de production : $\mathrm{J}_{\mathrm{T}}=\mathrm{Q} / \mathrm{p} \cdot \mathrm{z}$;

- capacité de traitement : $\mathrm{J}_{0}=\mathrm{Q}_{0} / \mathrm{p} . \mathrm{z}$;

- flux moyen de pervaporat : $\left\langle\mathrm{J}_{\mathrm{p}}\right\rangle=\mathrm{J}_{0}-\mathrm{J}_{\mathrm{T}}$;

- composition moyenne du pervaporat : $\left\langle\mathrm{C}^{\prime}\right\rangle=\left(\mathrm{J}_{0}, \mathrm{C}_{0}-\mathrm{J}_{\mathrm{T}}\right.$. $\left.\mathrm{C}_{\mathrm{T}}\right) /<\mathrm{J}_{\mathrm{p}}>$;

- rendement en produit épuré : $\mathrm{R}=\mathrm{Q}_{\mathrm{T}}\left(1-\mathrm{C}_{\mathrm{T}}\right) / \mathrm{Q}_{0}\left(1-\mathrm{C}_{0}\right)$;

- énergie consommée par $\mathrm{Kg}$ de produit épuré obtenu : $\mathrm{E}=\left\langle\mathrm{J}_{\mathrm{p}}\right\rangle .\left\langle\mathrm{L}_{\mathrm{p}}\right\rangle / \mathrm{J}_{\mathrm{T}} .\left\langle\mathrm{L}_{\mathrm{p}}\right\rangle$ représente la chaleur moyenne de vaporisation du pervaporat.

L'observation de la relation (2) montre que le débit $\mathrm{Q}$ en liquide purifié est proportionnel au produit : $\mathrm{J}_{\mathrm{p}}(\mathrm{C}) \cdot[6(\mathrm{C})-1]=\mathrm{K}(\mathrm{C})$.

Cette grandeur, que nous appellerons "critère de performance ", permet de chiffrer la qualité d'une membrane vis-à-vis d'une composition donnée, $\mathrm{C}$, et permet également de choisir la membrane la plus adaptée à un domaine de concentration donné. Par ailleurs, l'énergie électrique de pompage-recompression du pervaporat $\left\langle\mathrm{J}_{\mathrm{p}}\right\rangle$ est calculée à l'aide des données de Muller et Duquennoy, 1982.

\section{Bibliographie}

BINNing (R. C.), JAMES (F. E.) (1958). - How to separate by membrane permeation. Petroleum Refiner, 39, 214-215.

BLACK (C.) (1980). - Distillation modeling of ethanol recovery and dehydration processes for ethanol and gasohol. Chem. Eng. Prog., 76 (9), 78-85.

Hoover (K. C.), HWang (S. T.) (1982). - Pervaporation by a continuous membrane column. J. Membrane Sci., 10, 253-271.

LARKin (J. A.), Pemberton (R. C.) (1976). - Thermodynamic properties of mixtures of water and ethanol between 298.15 and $383.15 \mathrm{~K}$. National Physical Laboratory, Report Chem. 43, U.S.A.

LEEPER (S. A.), WANKAT (P.C.) (1982). - Gasohol production by extraction of ethanol from water using gasoline as solvent. Ind. Eng. Chem. Process Des. Dev., 21, 331-334.

Mehta (G. D.) (1982). - Comparison of membrane processes with distillation for alcohol/water separation. J. Membrane Sci., 12, 1-26.

Muller, Duquennoy (1982). - La recompression mécanique des vapeurs en distillation éthanol/eau. Informations Chimie, 222, 101-104.

SCHISSEL (P.), ORTH (R. A.) (1984). - Separation of ethanol-water mixtures by pervaporation through thin, composite membranes. J. Membrane Sci., 17, $109-120$.

SoukUP (P.B.). - Aufkonzentrierung und Absolutierung von Ethanol aus wässrigen Mischungen durch Pervaporation, Sept. 1983. Symposium on Synthetic membranes in Science and Industry, Tübingen. 